\title{
A Particle Swarm Optimization Approach for Optimum Design of PID Controller for nonlinear systems
}

\author{
Taeib Adel \\ Research Unit on Control, \\ Monitoring and Safety of Systems (C3S) \\ High School ESSTT \\ Email: taeibadel@live.fr
}

\author{
Chaari Abdelkader \\ Research Unit on Control, \\ Monitoring and Safety of Systems (C3S) \\ High School ESSTT \\ Email: abdelkader.chaari@yahoo.fr
}

\begin{abstract}
In this paper,a novel design method for determining the optimal proportional-integral-derivative (PID) controller parameters for Takagi-Sugeno fuzzy model using the particle swarm optimization (PSO) algorithm is presented. In order to assist estimating the performance of the proposed PSO-PID controller,a new timedomain performance criterion function has been used. The proposed approach yields better solution in term of rise time, settling time,maximum overshoot and steady state error condition of the system.the proposed method was indeed more efficient and robust in improving the step response.
\end{abstract}

\section{INTRODUCTION}

During the past decades, the process control techniques in the industry have made great advances. Numerous control methods such as adaptive control,neural control,and fuzzy control have been studied [1][2].(93-103). Among them,proportional-Integral-Derivative (PID) controllers have been widely used for speed and position control of various applications. Among the conventional PID tuning methods, the ZieglerNichols method [3] may be the most well known technique,but,In general,it is often hard to determine optimal or near optimal PID parameters with the Ziegler-Nichols formula in many industrial plants [4][5]. For these reasons,People have made lots of research, and proposed some advanced PID control methods,such as expert PID control based on knowledge inference[6],self-learning PID control based on regulation, neural network PID control based on connection mechanism[7],and intelligent PID control based on fuzzy logic[8,9]. Genetic algorithm (GA) has (566) been applied to self-tuning of PID parameters, too [10]. However,GA has the disadvantages of premature and slow convergence rate,and the need to set up many parameters. Recently,the computational intelligence has proposed particle swarm optimization (PSO) $[11,12]$ as opened paths to a new generation of advanced process control. The PSO algorithm, proposed by Kennedy and Eberhart [11] in 1995,was an evolution computation technology based on population intelligent methods. In comparison with genetic algorithm,PSO is simple,easy to realize and has very deep intelligent background. It is not only suitable for scientific research,but also suitable for engineering applications in particular. Thus,PSO received widely attentions from evolution computation field and other fields. Now the PSO has become a hotspot of research. Various objective functions based on error performance criterion are used to evaluate the performance of PSO algorithms. Each objective function is fundamentally the same except for the section of code that defines the specific error performance criterion being implemented to optimize the performance of a PID controlled system. Performance indices used to estimate the best parameters of PID controller are given by: $I S E, M S E$, and $I A E$. The main aim of this research paper is to establish a methodology for optimal design of PID controllers for Takagi-Sugeno (T-S) fuzzy model. the T-S fuzzy model is widely used in many research areas because of its excellent ability of nonlinear system description. It has a great capacity to approximate any nonlinear system [9]. for this, a particle swarm optimization (PSO) algorithm are proposed to improve controller by adjusting transfer function parameters. The rest of the paper is organized as follows. In section 2, a brief review of the TS fuzzy model formulation is given. In section 3,describes the standard PSO.PID controller design by the proposed PSO algorithm is described in Section 4. Some simulation results is shown in Section 5. Finally,some conclusions are made in section 6 .

\section{T-S FUZZY MODEL OF NONLINEAR SYSTEM}

We consider a class of nonlinear systems defined by:

$$
y(k+1)=f(x(k))
$$

With the regression vector represented by:

$x(k)=[y(k), y(k-1), \ldots, y(k-n), u(k), u(k-1), \ldots, u(k-n)]$

Here, $\mathrm{k}$ denotes the discrete time, and $\mathrm{n}$ define the number of delayed output. Through this contribution, the unknown function $f(x(k))$ is approximated by a T-S fuzzy model which is charities by rule consequents that are linear function of the input variables [13]. The rule base comprises $r$ rules of the form: 


$$
\begin{aligned}
& R^{i}: \text { if } x_{1} i s A_{1}^{i} \text { and if } x_{s} \text { is } A_{s}^{i} \text { then } \\
& y(k+1)=a_{i 1} y(k)+\ldots+a_{i n} y(k-n) \\
& +b_{i 1} u(k)+\ldots+b_{i n} u(k-n)
\end{aligned}
$$

Where $R^{i}$ denotes the $i^{\text {th }}$ fuzzy inference rule:

- $r$ is the number of inference rules;

- $A_{j}^{i}(j=1 \ldots s)$ are fuzzy sets;

- $u(k)$ is the system input variable;

- $y(k)$ is the system output;

- $a_{i 1}, \ldots, a_{i n}, b_{i 1}, \ldots, b_{i n}$ are coefficient of the $i^{t h}$ subsystem;

- $x(k)=\left[x_{i}, \ldots, x_{s}\right]$ are some measurable system variables. Let $\mu_{i}(x(k))$ be the normalized membership function of the inferred fuzzy set $A^{i}$, where $A^{i}=\prod_{j=1}^{s} A_{j}^{i}$ and $\sum_{i=1}^{r} \mu_{i}=1$.The output of T-S fuzzy model is computed:

$$
\begin{aligned}
y(k)= & \sum_{i=1}^{r} \mu_{i}\left[a_{i 1} y(k)+\ldots+a_{i n} y(j k-n)\right. \\
& \left.+b_{i 1} u(k)+\ldots+b_{i n} u(k-n)\right]
\end{aligned}
$$

\section{ESTIMATION METHOD OF RECURSIVE LEAST SQUARES}

$$
\text { (RLS) }
$$

For nonlinear systems the online adaptation is necessary to obtain a model able to continue the system in its evolution. The system described by can also be rewritten as:

$$
y(k)=\theta^{t} \Phi(k-1)
$$

This is a regression form, with $\theta$ being a system parameter vector and $\Phi$ a regression vector. It should be noted that the system (5) is in general nonlinear but it is linear with respect to its unknown parameter vectors. Based on parameterization (5), the identification algorithm giving estimates $\widehat{\theta}(k)$ of $\theta(k)$ can be obtained using the normalized least-squares algorithm [14]. We define:

$$
\begin{aligned}
& \varphi_{i}(k-1)=\left[\mu_{i} y(k-1) \ldots \mu_{i} y(k-n)\right. \\
& \left.\mu_{i} u(k-1) \ldots \mu_{i} u(k-n) \mu_{i}\right] \\
& \theta_{i}=\left[a_{i 1} \ldots a_{i n} b_{i 1} \ldots b_{i n} c_{i}\right] \\
& \varphi(k-1)=\left[\varphi_{1}^{t}(k-1) \varphi_{2}^{t}(k-1) \ldots \varphi_{r}^{t}(k-1)\right]^{t}
\end{aligned}
$$

The system described by (4) can also be rewritten as:

$$
P_{i}(k)=P_{i}(k-1)-\frac{P_{i}(k-1) \varphi_{i} \varphi_{i}^{t}(k) P_{i}(k-1)}{1+\varphi_{i}^{t}(k) P_{i}(k-1) \varphi_{i}(k-1)}
$$

\section{PID CONTROLLER}

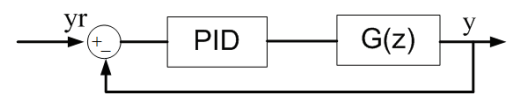

Fig. 1. A common feedback control system

The feedback control system is illustrated in Fig. 1 where yr,y are respectively the reference and controlled variables. The PID controller is used to improve the dynamic response as well as to reduce or eliminate the steady-state error. The derivative controller adds a finite zero to the open-loop plant transfer function and improves the transient response[15]. The integral controller adds a pole at the origin, thus increasing system type by one and reducing the steady-state error due to a step function to zero. The PID controller transfer function is

$$
C(z)=k p+k i \frac{z}{z-1}+k d \frac{z-1}{z}
$$

where $k p, k i$ and $k d$ are respectively the proportional, integral and derivative gains parameters of the PID controllers. We can also rewrite as Controller design attempts to minimize the system error produced by certain anticipated inputs[17]. The system error is defined as the difference between the desired response of the system and its actual response. Performance criteria are mainly based on measures of the system error. Basically, PID controller design method using criterion as tabulate in TABLE I.

A disadvantage of the $I A E$ and $I S E$ criteria is that its

TABLE I

PERFORMANCE ESTIMATION OF PID CONTROLLER

\begin{tabular}{|c||c|}
\hline Name of Criterion & Formula \\
\hline Integral of the Absolute Error (IAE) & $I A E=|e(k)|$ \\
\hline Integral of Square Error (ISE) & $I S E=\sum e(k)^{2}$ \\
\hline Integral of Time weighted Square Error (ITSE) & $I T S E=\sum k * e(k)^{2}$ \\
\hline
\end{tabular}

minimization can result in a response with relatively small overshoot but a long settling time because IAE and ISE performance criterion weights all errors equally independent of time. Although the ITSE performance criterion weights errors with time, the derivation processes of the analytical formula are complex and time consuming. In this paper,a new performance criterion in the time domain is proposed for evaluating the PID controller. A set of good control parameters $k p, k i$ and $k d$ can yield a good step response that will result in performance criteria minimization in the time domain. These performance criteria in the time domain include the overshoot $M_{p}$,rise time $T_{r}$, settling time $T_{s}$, and steady-state error $E_{s s}$. Therefore, a new performance criterion is defined as follows: time and settling time.

$$
W(k)=(1-\exp (-\beta))\left(M_{p}+E_{s s}\right)+(\exp (-\beta))\left(T_{s}-T_{r}\right)
$$

where $K=\left[\begin{array}{lll}k p & k i & k d\end{array}\right]$ and $\beta$ is the weighting factor.

\section{A. PARTICLE SWARM OPTIMIZATION}

Particle Swarm Optimization, first introduced by Kennedy and Eberhart,is one of optimization algorithms. It was developed through simulation of simplified social system, and has been found to be robust in solving continuous nonlinear optimization problems [16]. The PSO technique can generate a high quality solution within shorter calculation time and stable convergence characteristic than other stochastic methods. PSO is a population based search process where individuals, referred to as particles, are grouped into a swarm. 
Each particle in swarm represents a candidate solution to the optimization problem. In PSO technique,each particle is flown through the multidimensional search space,adjusting its position in search space according to its own experience and that of neighboring particles. A particle therefore makes use of best position encountered by itself and that of its neighbors to position itself toward an optimal solution. The effect is that particles fly toward a minimum,while still searching a wide area around the best solution. The performance of each particle (i.e., the closeness of a particle to a global optimum) is measured using a predefined fitness function, which encapsulates the characteristics of the optimization problem. As example, the $i^{t h}$ particle is represented as $x_{i}=$ $\left(x_{i, 1}, x_{i, 2}, \ldots, x_{i, d}\right)$ in the gdimensional space. The best previous position of the $j^{\text {th }}$ particle is recorded and represented as Pbesti $=\left(\right.$ pbest $_{i, 1}$, pbest $_{i, 2}, \ldots$, pbest $\left._{i, d}\right)$. The index of best particle among all particles in the group is represented by the gbestd. The rate of the position change (velocity) for particle $\mathrm{j}$ is represented as $v_{i}=\left(v_{i, 1} v_{i, 2}, \ldots, v_{i, d}\right)$.The modified velocity and position of each particle can be calculated using the current velocity and distance from pbest $_{i, d}$ to gbestd as shown in the following formulas:

$$
\begin{gathered}
v_{i d}(k+1)=w v_{i d}(k)+r_{1} * c_{1}\left(\text { pbest }_{i d}(k)-x_{i d}(k)\right) \\
+r_{2} * c_{2}\left(\text { gbest }_{g d}(k)-x_{i d}(k)\right) \\
x_{i d}(k+1)=x_{i d}(k)+v_{i d}(k+1)
\end{gathered}
$$

Where:

- pbest $_{i}$ is pbest of particle $i$.

- gbest $_{g}$ is gbest of the group.

- $r_{1}, r_{2}$ are two random numbers in the interval $[0,1]$.

- $c_{1}, c_{2}$ are positive constants,

- $w$ is the Inertia weight,is a parameter used to control the impact of the previous velocities on the current velocity. It influences the tradeoff between the global and local exploitation abilities of the particles. Weight is updated as $\omega=\omega_{\max }-\left(\frac{\omega \max -\omega_{\min }}{\text { iter } \max }\right)$ iter where $\omega_{\min }, \omega_{\max } t$ and iter $_{\max }$ are minimum,maximum values of $\omega_{i}$ ter, the current iteration number,and pre-specified maximum number of iteration cycles,respectively.

\section{B. IMPLEMENTATION OF A PSO-PID CONTROLLER}

In this paper, a PID controller using the PSO algorithm was developed to improve the step transient response of nonlinear system. It was also called the PSO-PID controller. The PSO algorithm was mainly utilized to determine three optimal controller parameters, and such that the controlled system could obtain a good step response output. For our case of design,we had to tune all the three parameters of PID such that it gives the best output results or in other words we have to optimize all the parameters of the PID for best results. Here we define a three dimensional search space in which all the three dimensions represent three different parameters of the PID. Each particular point in the search space represent a particular combination of $\left[\begin{array}{lll}k p & k i & k d\end{array}\right]$ for which a particular response is obtained The performance of the point or the combination of PID parameters is determined by a fitness function or the cost function. This fitness function consists of several component functions which are the performance index of the design. The point in the search space is the best point for which the fitness function attains an optimal value. For the case of our design,we have taken four component functions to define fittness function. The fittness function is a function of steady state error, peak overshoot, rise time and settling time. However the contribution of these component functions towards the original fittness function is determined by a scale factor that depends upon the choice of the designer. For this design the best point is the point where the fitness function has the minimal value.

\section{Proposed PSO-PID Controller}

the PSO-PID controller for searching the optimal controller parameters, $K p k i$, and , $k d$ with the PSO algorithm. Each individual $K$ contains three members $k p, k i$ and $k d$. Its dimension is $n * 3$. The searching procedures of the proposed PSO-PID controller were shown as below.

Step 1

- Specify the lower and upper bounds of the three controller parameters and initialize randomly the individuals of the population including searching points, velocities, pbest, and gbest.

- Determine the lower bound and the upper Bound $V_{d}^{\max }, V_{d}^{\min }, K_{d}^{\max }$ and $K_{d}^{\min }$.

step 2

- Evaluate the objective criterion and calculate the values of the four performance criteria $M_{p}, E_{s s}, t_{r}$ and $t_{s}$

step 3

- Compare the individual fitness of each particle to its previous gbest. If the fitness is better, update the fitness as gbest.

step 4

- Modify the velocity $v$ of each individual $K$ according to (8).

step 5

- if $v_{i d}^{k+1} \succ V_{d}^{\max }$,then $v_{j d}^{k+1}=V_{d}^{\max }$

- if $v_{i d}^{i d+1} \prec V_{d}^{\text {min }}$,then $v_{i d}^{k^{d}+1}=V_{d}^{\text {min }}$

Step 6

- Modify the member position of each individual $K$ according to (9).such that $K_{d}^{\min } \preceq K_{i d}^{k+1} \preceq K_{d}^{\max }$

step 7

- If the number of iterations reaches the maximum, then go to Step 8. Otherwise, go to Step 2.

Step 8

- The individual that generates the latest is an optimal controller parameter. 


\section{SIMULATION RESULTS}

This section presents a simulation example to shown an application of the proposed control algorithm and its satisfactory performance. The nonlinear system is written as the following recursive form:

$$
\begin{aligned}
y(k)=a_{1}^{\prime} & \sin (y(k-1))+a_{2}^{\prime} y(k-2)+a_{3}^{\prime} u(k-2) y(k-3) \\
& +b_{1}^{\prime} u(k-1)+b_{2}^{\prime}\left(\tanh \left(0.7 u(k-3)^{2}\right)\right)
\end{aligned}
$$

With $a_{1}^{\prime}=0.4, a_{2}^{\prime}=0.3, a_{3}^{\prime}=0.1, b_{1}^{\prime}=0.6$ and $b_{2}^{\prime}=1.8$. The input signal applied to plant (14) is a finite sequence of uniformly distributed random variables with range $[-2,2]$. The consequent parameters of each rule Takagi-Sugeno fuzzy model are computed from equation (5) and adapted by using $R L S$ algorithm with for gueting factor $\lambda=0.9$ For the rule $i$ :

$$
\begin{gathered}
R_{i}: \text { if } y(k-1) \text { is } A_{i}, \text { and } y(k-2) \text { is } B_{i} \\
\text { and } y(k-3) \text { is } C_{i},, \text { and } u(k-1) \text { is } D_{i} \\
\text { and } u(k-2) \text { is } I_{i} \\
\text { then } y_{i}(k)=-a_{i 1} y(k-1)-a_{i 2} y(k-2)+-a_{i 3} y(k-3) \\
+b_{i 1} u(k-1)+b_{i 2} u(k-2)
\end{gathered}
$$

The system response is shown in figure(1), the proposed method can guarantee a good control performance.

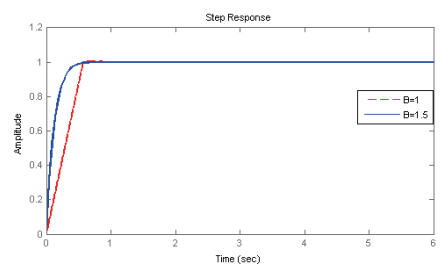

Fig. 2. system response

TABLE II

PERFORMANCE ESTIMATION OF PID CONTROLLER

\begin{tabular}{|c||c||c|}
\hline$\beta$ & 1 & 1.5 \\
\hline Swarm size & 100 & 100 \\
\hline $\mathrm{kp}$ & 9.6853 & 7.5342 \\
\hline $\mathrm{ki}$ & 3.9095 & 2.4439 \\
\hline $\mathrm{kd}$ & 9.1067 & 8.2874 \\
\hline overshoot & 0.2180 & 0.0250 \\
\hline rise time & 0.4595 & 0.4635 \\
\hline settling time & 0.5629 & 0.5678 \\
\hline
\end{tabular}

\section{CONCLUSIONS}

This paper presents a novel design method for determining the PID controller parameters using the PSO method for Takagi-Sugeno fuzzy model. The proposed method integrates the PSO algorithm with the new performance criterion into a PSO-PID controller. Through the simulation, the results show that the proposed controller can perform an efficient search for the optimal PID controller parameters.

\section{REFERENCES}

[1] A. visioli, Tuning of PID controllers with fuzzy logic, proc. Inst. Elec, proc. Inst. Elec. Eng. contr. theory applicat-vol, 148. no-1, pp-1-8, jan. 2008.

[2] T. Kawabe and T. Tagami,A real coded genetic algorithm for matrix inequality design approach of robust PID controller with two degrees of freedom, in Proc. $12^{\text {th }}$ IEEE Int. Symp. Intell. Contr, Istanbul, Turkey, July 1997, pp. 119124. 1993.

[3] K Ogata, Modern Control Systems, University of Minnesota, Prentice Hall, 1987.

[4] A. Visioli,Tuning of PID controllers with fuzzy logic,Proc. Inst. Elect. Eng. Contr. Theory Applicat, vol. 148, no. 1, pp. 18, Jan. 2001. Conference, Vol. 1, 1999.

[5] R. A. Krohling and J. P. Rey, Design of optimal disturbance rejection PID controllers using genetic algorithm, IEEE Trans. Evol, Comput, vol. 5, pp. 7882, Feb. 2001.

[6] Conradie A, Miikkulainen R, and Aldrich C, Adaptive Control Utilizing Neural Swarming, In Proceedings of the Genetic and Evolutionary Computation Conferences, USA, 2002

[7] Hossein Shayeghi, Heidar Ali Shayanfar and Aref Jalili, Multi Stage Fuzzy PID Load Frequency Controller in a Restructured Power System, Journal of Electrical Engineering, Vol. 58, No. 2, pp. 61-70, 2007.

[8] Saban Cetin, and Ozgr Demir, Fuzzy PID Controller with Coupled Rules for a Nonlinear Quarter Car Model, World Academy of Science, Engineering and Technology Vol. 41, pp. 238-241, 2008.

[9] Aye Aye Mon, Fuzzy Logic PID Control of Automatic Voltage Regulator System, Proceedings of PWASET, Vol. 38, Feb, 2009.

[10] Cipperfield A. Flemming P, and Fonscea C, Genetic Algorithms for Control System Engineering, in Proceedings Adaptive Computing in Engineering Design Control, pp- 128-133,1994.

[11] Kennedy J. and Eberhart C, Particle Swarm Optimization, Proceedings of the IEEE International Conference on Neural Networks, Australia, pp. 1942-1948,1995.

[12] Oliveira, P. M, Cunha, J. B, and Coelho,J.o.P,Design of PID controllers using the Particle Swarm Algorithm, Twenty-First IASTED International Conference: Modeling, Identification, and Control (MIC 2002), Innsbruck, Austria. 2002

[13] I. Lagrat, H. Ouakka, and I. Boumhidi,Adaptive control of a class of nonlinear systems based on Takagi-Sugeno fuzzy model, ICTIS 07, Fez, Morocco, 3-5 April 2007.

[14] L. Praly, Robustness of indirect adaptive control based on pole placement design. Proc, Of the 1st IFAC Workshop on adaptive systems in control and signal processing, San Francisco, USA.1983.

[15] Mahmud Iwan Solihin, Lee Fook Tack and Moey Leap Kean,Tuning of PID Controller Using Particle Swarm Optimization (PSO), School of Engineering, UCSI University No. 1, Jalan Menara Gading, UCSI Heights, 56000. 14 - 15 January 2011.

[16] S.J.Qin \& T.A.Badgwell, A survey of industrial model predictive control technology, Control Engineering Practice, No. 11, pp733- 764, 2003.

[17] S. Morkos, H. Kamal,Optimal Tuning of PID Controller using Adaptive Hybrid Particle Swarm Optimization Algorithm,Int. J. of Computers, Communications and Control, ISSN 1841-9836, 2012. 